\title{
Existence and global exponential stability of periodic solutions for $n$-dimensional neutral dynamic equations on time scales
}

\author{
Bing $\mathrm{Li}^{1}$, Yongkun $\mathrm{Li}^{1 *}$ and Xuemei Zhang ${ }^{2}$
}

*Correspondence:

yklie@ynu.edu.cn

${ }^{1}$ Department

of Mathematics, Yunnan

University, Kunming 650091,

Yunnan, People's Republic

of China

Full list of author information is available at the end of the article

\begin{abstract}
In this paper, by using the existence of the exponential dichotomy of linear dynamic equations on time scales and the theory of calculus on time scales, we study the existence and global exponential stability of periodic solutions for a class of $n$-dimensional neutral dynamic equations on time scales. We also present an example to illustrate the feasibility of our results. The results of this paper are completely new and complementary to the previously known results even in both the case of differential equations (time scale $\mathbb{T}=\mathbb{R}$ ) and the case of difference equations (time scale $\mathbb{T}=\mathbb{Z}$ ).
\end{abstract}

Keywords: Periodic solution, Neutral delay, Exponential stability, $n$-Dimensional neutral dynamic equations, Time scale

Mathematics Subject Classification: 34K13, 34K20, 34K40, 34N05

\section{Background}

The theory of dynamic equations on time scales was introduced by Hilger (1990) in 1988 in order to unify the study of continuous and discrete calculus. Since then, the study on dynamic equations on time scales has received much attention of many scholars. For example, in DaCunha (2005), the author studied the stability of the following linear dynamic equation on time scales:

$$
x^{\Delta}(t)=A(t) x(t), x\left(t_{0}\right)=x_{0}, \quad t_{0} \in \mathbb{T} .
$$

In $\mathrm{Du}$ and Tien (2007), the authors obtained some conditions ensuring the stability of the trivial solution for the following dynamic equation on time scales:

$$
x^{\Delta}(t)=A(t) x(t)+f(t, x), \quad t \in \mathbb{T} .
$$

For other studies on dynamic equations on time scales, we refer the reader to Bohner and Peterson (2001), Graef and Hill (2015), Li and Sun (2013), Li and Xu (2011), Lupulescu and Younus (2011), Su and Feng (2014), Wang et al. (2010), Zhang et al. (2010a, b, 2014), Zhou and $\mathrm{Li}(2010,2012)$ and the references therein.

Since it is natural and important that systems will contain some information about the derivative of the past state to further describe and model the dynamics for such complex

(C) $2016 \mathrm{Li}$ et al. This article is distributed under the terms of the Creative Commons Attribution 4.0 International License (http:// creativecommons.org/licenses/by/4.0/, which permits unrestricted use, distribution, and reproduction in any medium, provided you give appropriate credit to the original author(s) and the source, provide a link to the Creative Commons license, and indicate if changes were made. 
reactions, many authors have studied the existence of solutions of various neutral delay models (Abbas and Bahuguna 2008; Ardjouni and Djoudi 2012; Chen and Lin 2010; Hovhannisyan 2014; Kaufmann and Raffoul 2006; Li and Saker 2014; Xu et al. 2007; Zhang et al. 2009). However, to the best of our knowledge, there are few papers published on the existence and stability of periodic solutions to neutral dynamic equations on time scales.

Motivated by the above discussion, in this paper, we are concerned with the following neutral dynamic equation on time scales:

$$
x^{\Delta}(t)=A(t) x(t)+f\left(t, x_{t}, x_{t}^{\Delta}\right), \quad t \in \mathbb{T},
$$

where $\mathbb{T}$ is an $\omega$-periodic time scale and satisfies that for $t, s \in \mathbb{T}, t+s \in \mathbb{T}$, $A(t)=\left(a_{i j}(t)\right)_{n \times n}$ is a regressive and rd-continuous matrix-valued function, $f \in C_{r d}\left(\mathbb{T} \times B C \times B C, \mathbb{R}^{n}\right)$ and $f\left(t, x_{t}, x_{t}^{\Delta}\right)$ is $\omega$-periodic whenever $x$ is a $\Delta$-differentiable $\omega$-periodic function with rd-continuous $\Delta$-derivative, where $B C$ denotes the Banach space of all bounded rd-continuous functions $\varphi:[-\theta, 0] \cap \mathbb{T} \rightarrow \mathbb{R}^{n}$ with the norm $|\varphi|_{0}=\max _{1 \leq i \leq n} \sup _{s \in[-\theta, 0] \cap \mathbb{T}}\left|\varphi_{i}(s)\right|$ where $\varphi=\left(\varphi_{1}, \phi_{2}, \ldots, \varphi_{n}\right)^{T}, \omega>0$ is a constant, $\theta$ is a positive number or $\infty$ and if $\theta=\infty$, then we set $[-\theta, 0]=(-\infty, 0]$. If $x, x^{\Delta} \in C_{r d}\left(\mathbb{T}, \mathbb{R}^{n}\right)$, then for any $t \in \mathbb{T}, x_{t}$ and $x_{t}^{\Delta} \in B C$ are defined by $x_{t}(s)=x(t+s)$ and $x_{t}^{\Delta}(s)=x^{\Delta}(t+s)$ for $s \in[-\theta, 0] \cap \mathbb{T}$, respectively.

Remark 1 Throughout this paper, we denote the class of all functions $f: \mathbb{T} \times B C \times B C \rightarrow \mathbb{R}^{n}$ that are rd-continuous with respect to their first argument and continuous with respect to their second and third arguments by $C_{r d}\left(\mathbb{T} \times B C \times B C, \mathbb{R}^{n}\right)$.

Remark 2 If $\theta$ is a finite positive number, then Eq. (1) is a bounded delay neutral dynamic equation on time scales and if $\theta$ is infinite then Eq. (1) is a unbounded delay neutral dynamic equation on time scales.

Our main purpose of this paper is to study the existence and global exponential stability of periodic solutions for (1) by using the exponential dichotomy of linear dynamic equations and the theory of calculus on time scales. As we all know, Eq. (1) contains many differential equation models and difference equation models as its special cases. For example, if we take

$$
\begin{aligned}
\mathbb{T}=\mathbb{R}, \quad A(t)= & \operatorname{diag}\left[-b_{1}(t),-b_{2}(t), \ldots,-b_{n}(t)\right], \quad f=\left(f_{1}, f_{2}, \ldots, f_{n}\right), \\
f_{i}\left(t, \varphi, \varphi^{\prime}\right)= & \sum_{j=1}^{n} a_{i j}(t) f_{j}\left(\varphi_{j}(0)\right)+\sum_{j=1}^{n} b_{i j}(t) f_{j}\left(\varphi_{j}\left(-\tau_{i j}(t)\right)\right) \\
& +c_{i} \varphi_{i}^{\prime}\left(-\sigma_{i}(t)\right)+I_{i}(t), \quad i=1,2, \ldots, n,
\end{aligned}
$$

where $\varphi=\left(\varphi_{1}, \varphi_{2}, \ldots, \varphi_{n}\right)$, then (1) reduces to the following neural network with neutral type delays:

$$
\begin{aligned}
x_{i}^{\prime}(t)= & -b_{i}(t) x_{i}(t)+\sum_{j=1}^{n} a_{i j}(t) f_{j}\left(x_{j}(t)\right)+\sum_{j=1}^{n} b_{i j}(t) f_{j}\left(x_{j}\left(t-\tau_{i j}(t)\right)\right) \\
& +c_{i} x_{i}^{\prime}\left(t-\sigma_{i}(t)\right)+I_{i}(t), \quad i=1,2, \ldots, n,
\end{aligned}
$$


which was studied in Li et al. (2012). If we take

$$
\mathbb{T}=\mathbb{R}, \quad A(t)=\operatorname{diag}\left[a_{1}(t), a_{2}(t), \ldots, a_{n}(t)\right],
$$

$$
f\left(t, \varphi, \varphi^{\prime}\right)=\lambda F\left(t, \varphi_{t}\right)
$$

then (1) reduces to

$$
x^{\prime}=A(t) x(t)+\lambda F\left(t, x_{t}\right),
$$

which was studied in Liu and $\mathrm{Li}$ (2004). Even in both the case of differential equations (time scale $\mathbb{T}=\mathbb{R}$ ) and the case of difference equations $(\mathbb{T}=\mathbb{Z}$ ), our results are completely new and complementary to the previously known results.

For convenience, we denote $[a, b]_{\mathbb{T}}=\{t \mid t \in[a, b] \cap \mathbb{T}\}$. For an rd-continuous $\omega$-periodic function $h: \mathbb{T} \rightarrow \mathbb{R}$, denote $h^{+}=\sup _{t \in[0, \omega]_{\mathbb{T}}}|h(t)|, h^{-}=\inf _{t \in[0, \omega]_{\mathbb{T}}}|h(t)|$. For an rd-continuous $\omega$-periodic function $u: \mathbb{T} \rightarrow \mathbb{R}^{n}$, we define $|u|_{0}=\max _{1 \leq i \leq n} \max _{t \in[0, \omega] \mathbb{T}}\left|u_{i}(t)\right|$. For matrices or vectors $A, B, A \geq B$ (or $A>B$ ) means that all entries of $A$ are greater than or equal to (or greater than) corresponding entries of $B$. For $A(t)=\left(a_{i j}(t)\right)_{n \times n}$, we can take ||$A \|=\max _{1 \leq i \leq n} \sum_{j=1}^{n}\left|a_{i j}^{+}\right|$.

The initial condition of (1) is

$$
x(s)=\phi(s), x^{\Delta}(s)=\phi^{\Delta}(s), \quad s \in[-\theta, 0]_{\mathbb{T}},
$$

where $\phi \in C_{r d}^{1}\left([-\theta, 0]_{\mathbb{T}}, \mathbb{R}^{n}\right)$.

Throughout this paper, we assume that the following condition holds:

$\left(H_{1}\right) \quad f \in C_{r d}\left(\mathbb{T} \times B C \times B C, \mathbb{R}^{n}\right)$ is $\omega$-periodic with respect to its first argument and there exist positive constants $L_{1}, L_{2}$ such that

$$
\left|f\left(t, \varphi_{1}, \psi_{1}\right)-f\left(t, \varphi_{2}, \psi_{2}\right)\right|_{0} \leq L_{1}\left|\varphi_{1}-\varphi_{2}\right|_{0}+L_{2}\left|\psi_{1}-\psi_{2}\right|_{0}
$$

for all $t \in \mathbb{T}$ and $\varphi_{i}, \psi_{i} \in B C, i=1,2$.

\section{Preliminaries}

In this section, we introduce some definitions and state some preliminary results.

Let $\mathbb{T}$ be a nonempty closed subset (time scale) of $\mathbb{R}$. The forward and backward jump operators $\sigma, \rho: \mathbb{T} \rightarrow \mathbb{T}$ and the graininess $\mu: \mathbb{T} \rightarrow \mathbb{R}^{+}$are defined, respectively, by

$$
\sigma(t)=\inf \{s \in \mathbb{T}: s>t\}, \quad \rho(t)=\sup \{s \in \mathbb{T}: s<t\} \quad \text { and } \quad \mu(t)=\sigma(t)-t .
$$

A point $t \in \mathbb{T}$ is called left-dense if $t>\inf \mathbb{T}$ and $\rho(t)=t$, left-scattered if $\rho(t)<t$, right-dense if $t<\sup \mathbb{T}$ and $\sigma(t)=t$, and right-scattered if $\sigma(t)>t$. If $\mathbb{T}$ has a left-scattered maximum $m$, then $\mathbb{T}^{k}=\mathbb{T} \backslash\{m\}$; otherwise $\mathbb{T}^{k}=\mathbb{T}$. If $\mathbb{T}$ has a right-scattered minimum $m$, then $\mathbb{T}_{k}=\mathbb{T} \backslash\{m\}$; otherwise $\mathbb{T}_{k}=\mathbb{T}$.

A function $f: \mathbb{T} \rightarrow \mathbb{R}$ is right-dense continuous provided it is continuous at rightdense points in $\mathbb{T}$ and its left-side limits exist at left-dense points in $\mathbb{T}$. If $f$ is continuous at each right-dense point and each left-dense point, then $f$ is said to be continuous on $\mathbb{T}$. We denote the class of all rd-continuous functions $f: \mathbb{T} \rightarrow \mathbb{R}$ by $C_{r d}(\mathbb{T}, \mathbb{R})$.

For $y: \mathbb{T} \rightarrow \mathbb{R}$ and $t \in \mathbb{T}^{k}$, we define the delta derivative of $y(t), y^{\Delta}(t)$, to be the number (if it exists) with the property that for a given $\varepsilon>0$, there exists a neighborhood $U$ of $t$ such that 


$$
\left|[y(\sigma(t))-y(s)]-y^{\Delta}(t)[\sigma(t)-s]\right|<\varepsilon|\sigma(t)-s|
$$

for all $s \in U$.

We denote the class of all $\Delta$-differentiable functions with rd-continuous $\Delta$-derivative $f: \mathbb{T} \rightarrow \mathbb{R}$ by $C_{r d}^{1}(\mathbb{T}, \mathbb{R})$.

If $y$ is continuous, then $y$ is right-dense continuous, and if $y$ is $\Delta$-differentiable at $t$, then $y$ is continuous at $t$.

Let $y$ be right-dense continuous. If $Y^{\Delta}(t)=y(t)$, then we define the delta integral by $\int_{a}^{t} y(s) \Delta s=Y(t)-Y(a)$. Assume that $f: \mathbb{T} \rightarrow \mathbb{R}^{n}$ is a function and $f(t)=\left(f_{1}(t), \ldots, f_{n}(t)\right)$, then we define $\int_{a}^{t} f(s) \Delta s=\left(\int_{a}^{t} f_{1}(s) \Delta s, \ldots, \int_{a}^{t} f_{n}(s) \Delta s\right)$ (provided it exists).

Definition 1 (Bohner and Peterson 2001) Let $A$ be an $m \times n$-matrix-valued function on $\mathbb{T}$. We say that $A$ is $r d$-continuous on $\mathbb{T}$ if each entry of $A$ is $r d$-continuous on $\mathbb{T}$. We say that $A$ is differentiable on $\mathbb{T}$ provided each entry of $A$ is differentiable on $\mathbb{T}$, and in this case we put $A^{\Delta}=\left(a_{i j}^{\Delta}\right)_{m \times n}$, where $A=\left(a_{i j}\right)_{m \times n}$.

Definition 2 (Kaufmann and Raffoul 2006) We say that a time scale $\mathbb{T}$ is periodic if there exists $p>0$ such that if $t \in \mathbb{T}$, then $t \pm p \in \mathbb{T}$. For $\mathbb{T} \neq \mathbb{R}$, the smallest positive $p$ is called the period of the time scale.

Definition 3 (Kaufmann and Raffoul 2006) Let $\mathbb{T} \neq \mathbb{R}$ be a periodic time scale with period $p$. We say that the function $f: \mathbb{T} \rightarrow \mathbb{R}$ is periodic with period $\omega$ if there exists a natural number $n$ such that $\omega=n p, f(t+\omega)=f(t)$ for all $t \in \mathbb{T}$ and $\omega$ is the smallest positive number such that $f(t+\omega)=f(t)$.

Definition 4 (Bohner and Peterson 2001) A $n \times n$-matrix-valued function $A$ on time scale $\mathbb{T}$ is called regressive (respect to $\mathbb{T}$ ) provided $I+\mu(t) A(t)$ is invertible for all $t \in \mathbb{T}^{k}$.

Definition 5 (Bohner and Peterson 2001) Let $A, B$ be two $n \times n$-matrix-valued regressive functions on $\mathbb{T}$, we define

$$
\begin{aligned}
& (A \oplus B)(t):=A(t)+B(t)+\mu(t) A(t) B(t), \\
& (\ominus A)(t):=-[I+\mu(t) A(t)]^{-1} A(t)=-A(t)[I+\mu(t) A(t)]^{-1}, \\
& (A(t)) \ominus(B(t)):=(A(t)) \oplus(\ominus(B(t)))
\end{aligned}
$$

for all $t \in \mathbb{T}^{k}$.

Definition 6 (Bohner and Peterson 2001) Let $t_{0} \in \mathbb{T}$ and assume that $A \in \mathcal{R}$ is a $n \times n$ -matrix-valued function. The unique matrix-valued solution of the initial value problem

$$
x^{\Delta}(t)=A(t) x(t), \quad x\left(t_{0}\right)=I,
$$

where, $I$ denotes as usual the $n \times n$-identity matrix, is called the matrix exponential function (at $\left.t_{0}\right)$ and it is denoted by $e_{A}\left(\cdot, t_{0}\right)$. 
Remark 3 Assume that $A$ is a constant $n \times n$-matrix. If $\mathbb{T}=\mathbb{R}$, then $e_{A}\left(t, t_{0}\right)=e^{A\left(t-t_{0}\right)}$, while if $\mathbb{T}=\mathbb{Z}$ and $I+A$ is invertible, then $e_{A}\left(t, t_{0}\right)=(I+A)^{\left(t-t_{0}\right)}$.

Lemma 1 (Bohner and Peterson 2001) Let $A \in \mathcal{R}$ be a $n \times n$-matrix-valued functions on $\mathbb{T}$ and suppose that $f: \mathbb{T} \rightarrow \mathbb{R}^{n}$ is $r d$-continuous. Let $t_{0} \in \mathbb{T}$ and $x_{0} \in \mathbb{R}^{n}$. Then the initial value problem

$$
x^{\Delta}(t)=A(t) x(t)+f(t), \quad x\left(t_{0}\right)=x_{0}
$$

has a unique solution $x: \mathbb{T} \rightarrow \mathbb{R}^{n}$, which is given by

$$
x(t)=e_{A}\left(t, t_{0}\right) x_{0}+\int_{t_{0}}^{t} e_{A}(t, \sigma(s)) f(s) \Delta s .
$$

Lemma 2 (Bohner and Peterson 2001) If $A, B \in \mathcal{R}$ are matrix-valued functions on $\mathbb{T}$, then

(i) $\quad e_{0}(t, s) \equiv I$ and $e_{A}(t, t) \equiv I$;

(ii) $\quad e_{A}(\sigma(t), s)=(I+\mu(t) A(t)) e_{A}(t, s)$;

(iii) $e_{A}(t, s)=e_{A}^{-1}(s, t)$;

(iv) $e_{A}(t, s) e_{A}(s, r)=e_{A}(t, r)$;

(v) $\quad e_{A}(t, s) e_{B}(t, s)=e_{A \oplus B}(t, s)$, if $e_{A}(t, s)$ and $B(t)$ commute.

Lemma 3 (Bohner and Peterson 2001) If $A \in \mathcal{R}$ and $a, b, c \in \mathbb{T}$, then

$$
\left[e_{A}(c, \cdot)\right]^{\Delta}=-\left[e_{A}(c, \cdot)\right]^{\sigma} A
$$

and

$$
\int_{a}^{b} e_{A}(c, \sigma(t)) A(t) \Delta t=e_{A}(c, a)-e_{A}(c, b) .
$$

Definition 7 (Zhang et al. 2010a) Let $x \in \mathbb{R}^{n}$ and $A(t)$ be $a n \times n$ matrix-valued function on $\mathbb{T}$, the linear system

$$
x^{\Delta}(t)=A(t) x(t), \quad t \in \mathbb{T}
$$

is said to admit an exponential dichotomy on $\mathbb{T}$ if there exist positive constants $k_{i}, \alpha_{i}, i=1,2$, projection $P$ and the fundamental solution matrix $X(t)$ of $(2)$ satisfying

$$
\begin{aligned}
\left|X(t) P X^{-1}(s)\right|_{0} \leq k_{1} e_{\ominus \alpha_{1}}(t, s), & s, t \in \mathbb{T}, t \geq s, \\
\left|X(t)(I-P) X^{-1}(s)\right|_{0} \leq k_{2} e_{\ominus \alpha_{2}}(s, t), & s, t \in \mathbb{T}, t \leq s .
\end{aligned}
$$

Lemma 4 (Zhang et al. 2010a) If (2) admits an exponential dichotomy, then the following $\omega$-periodic system:

$$
X^{\Delta}(t)=A(t) X(t)+g(t), \quad t \in \mathbb{T}
$$

has an $\omega$-periodic solution as follows:

$$
X(t)=\int_{-\infty}^{t} X(t) P X^{-1}(\sigma(s)) g(s) \Delta s-\int_{t}^{+\infty} X(t)(I-P) X^{-1}(\sigma(s)) g(s) \Delta s,
$$

where $X(t)$ is the fundamental solution matrix of $(2)$. 
Lemma 5 (Zhang et al. 2010a) If $A(t)$ is a uniformly bounded rd-continuous $n \times n$ matrix-valued function on $\mathbb{T}$ and there is a $\delta>0$ such that

$$
\left|a_{i i}(t)\right|-\sum_{j \neq i}\left|a_{i j}(t)\right|-\frac{1}{2} \mu(t)\left(\sum_{j=1}^{n}\left|a_{i j}(t)\right|\right)^{2}-\delta^{2} \mu(t) \geq 2 \delta, \quad t \in \mathbb{T}, i=1,2, \ldots, n,
$$

then (2) admits an exponential dichotomy on $\mathbb{T}$.

Definition 8 Let $x(t)$ be an $\omega$-periodic solution of (1) with initial value $\varphi(s)$. If there exists a positive constant $\lambda$ with $-\lambda \in \mathcal{R}^{+}$such that for $t_{0} \in[-\theta, 0]_{\mathbb{T}}$, there exists $M>1$ such that for an arbitrary solution $y(t)$ of (1) with initial value $\psi(s)$ satisfies

$$
\|y-x\|_{\mathbb{X}} \leq M\|\varphi-\psi\|_{\mathbb{X}} e_{\ominus \lambda}\left(t, t_{0}\right), \quad t \in[-\theta, \infty)_{\mathbb{T}}, t \geq t_{0} .
$$

Then the solution $x(t)$ is said to be globally exponentially stable.

\section{Existence of periodic solutions}

Set $\mathbb{X}=\left\{\varphi \in C_{r d}^{1}\left(\mathbb{T}, \mathbb{R}^{n}\right) \mid \varphi\right.$ is $\omega$-periodic on $\left.\mathbb{T}\right\}$ with the norm $\|\varphi\|_{\mathbb{X}}=\max \left\{|\varphi|_{0},\left|\varphi^{\Delta}\right|_{0}\right\}$, where $|\varphi|_{0}=\max _{1 \leq i \leq n} \sup _{t \in[0, \omega]_{\mathbb{T}}}\left|\varphi_{i}(t)\right|,\left|\varphi^{\Delta}\right|_{0}=\max _{1 \leq i \leq n} \sup _{t \in[0, \omega] \mathbb{T}}\left|\varphi_{i}^{\Delta}(t)\right|$, then $\mathbb{X}$ is a Banach space.

\section{Theorem 1 Let $\left(H_{1}\right)$ hold. Suppose that}

$\left(H_{2}\right)$ system (2) admits an exponential dichotomy on $\mathbb{T}$ with constants $k_{i}, \alpha_{i}, i=1,2$;

$\left(H_{3}\right) \quad q=: \max \left\{\frac{k_{1}\left(1+\vartheta \alpha_{1}\right)}{\alpha_{1}}+\frac{k_{2}}{\alpha_{2}},\|A\|\left(\frac{k_{1}\left(1+\vartheta \alpha_{1}\right)}{\alpha_{1}}+\frac{k_{2}}{\alpha_{2}}\right)+1\right\}\left(L_{1}+L_{2}\right)<1$, where $\vartheta=\sup _{t \in \mathbb{T}} \mu(t)$. Then (1) has a unique $\omega$-periodic solution.

Proof By $\left(H_{3}\right)$, we can take a positive constant $L$ satisfying

$$
\max \left\{\frac{k_{1}\left(1+\vartheta \alpha_{1}\right)}{\alpha_{1}}+\frac{k_{2}}{\alpha_{2}},\|A\|\left(\frac{k_{1}\left(1+\vartheta \alpha_{1}\right)}{\alpha_{1}}+\frac{k_{2}}{\alpha_{2}}\right)+1\right\}\left(\left(L_{1}+L_{2}\right) L+a\right) \leq L,
$$

where $a=|f(\cdot, 0,0)|_{0}$. We set $\mathbb{X}_{0}=\left\{\varphi \in \mathbb{X} \mid\|\varphi\|_{\mathbb{X}} \leq L\right\}$. For any given $\varphi \in \mathbb{X}_{0}$, we consider the following periodic system:

$$
x^{\Delta}(t)=A(t) x(t)+f\left(t, \varphi_{t}, \varphi_{t}^{\Delta}\right) .
$$

Since $\left(H_{2}\right)$ holds, by Lemma 4 , we obtain that (3) has an $\omega$-periodic solution, which is expressed as follows:

$x^{\varphi}(t)=\int_{-\infty}^{t} X(t) P X^{-1}(\sigma(s)) f\left(s, \varphi_{s}, \varphi_{s}^{\Delta}\right) \Delta s-\int_{t}^{+\infty} X(t)(I-P) X^{-1}(\sigma(s)) f\left(s, \varphi_{s}, \varphi_{s}^{\Delta}\right) \Delta s$.

For $\varphi \in \mathbb{X}_{0}$, define the following operator:

$$
\Phi: \mathbb{X}_{0} \rightarrow \mathbb{X}_{0}, \varphi \rightarrow x^{\varphi} .
$$

First we show that for any $\varphi \in \mathbb{X}_{0}$, we have $\Phi \varphi \in \mathbb{X}_{0}$. Note that 


$$
\begin{aligned}
\left|f\left(s, \varphi_{s}, \varphi_{s}^{\Delta}\right)\right|_{0} & \leq\left|f\left(s, \varphi_{s}, \varphi_{s}^{\Delta}\right)-f(s, 0,0)\right|_{0}+|f(s, 0,0)|_{0} \\
& \leq L_{1}|\varphi|_{0}+L_{2}\left|\varphi^{\Delta}\right|_{0}+a \\
& \leq\left(L_{1}+L_{2}\right)\|\varphi\|_{\mathbb{X}}+a .
\end{aligned}
$$

So, we have that

$$
\begin{aligned}
|\Phi \varphi|_{0}= & \mid \int_{-\infty}^{t} X(t) P X^{-1}(\sigma(s)) f\left(s, \varphi_{s}, \varphi_{s}^{\Delta}\right) \Delta s \\
& -\left.\int_{t}^{+\infty} X(t)(I-P) X^{-1}(\sigma(s)) f\left(s, \varphi_{s}, \varphi_{s}^{\Delta}\right) \Delta s\right|_{0} \\
\leq & \sup _{t \in[0, \omega]_{\mathbb{T}}}\left(\int_{-\infty}^{t}\left|X(t) P X^{-1}(\sigma(s))\right|_{0}\left|f\left(s, \varphi_{s}, \varphi_{s}^{\Delta}\right)\right|_{0} \Delta s\right. \\
& \left.+\int_{t}^{+\infty}\left|X(t)(I-P) X^{-1}(\sigma(s))\right|_{0}\left|f\left(s, \varphi_{s}, \varphi_{s}^{\Delta}\right)\right|_{0} \Delta s\right) \\
\leq & \left(\left(L_{1}+L_{2}\right)\|\varphi\|_{\mathbb{X}}+a\right)\left(\sup _{t \in[0, \omega] \mathbb{T}}\left|\int_{-\infty}^{t} k_{1} e_{\ominus \alpha_{1}}(t, \sigma(s)) \Delta s\right|\right. \\
& \left.+\sup _{t \in[0, \omega]]_{\mathbb{T}}}\left|\int_{t}^{+\infty} k_{2} e_{\ominus \alpha_{2}}(\sigma(s), t) \Delta s\right|\right) \\
= & \left(\left(L_{1}+L_{2}\right)\|\varphi\|_{\mathbb{X}}+a\right)\left(\sup _{t \in[0, \omega]_{\mathbb{T}}}\left|k_{1} \int_{-\infty}^{t}\left(1+\mu(s) \alpha_{1}\right) e_{\alpha_{1}}(s, t) \Delta s\right|\right. \\
& \left.+\sup _{t \in[0, \omega]_{\mathbb{T}}}\left|k_{2} \int_{t}^{+\infty}\left(1+\mu(s) \ominus \alpha_{2}\right) e_{\ominus \alpha_{2}}(s, t) \Delta s\right|\right) \\
= & \left(\left(L_{1}+L_{2}\right)\|\varphi\|_{\mathbb{X}}+a\right)\left(\frac{k_{1}\left(1+\vartheta \alpha_{1}\right)}{\alpha_{1}} \sup _{t \in[0, \omega] \mathbb{T}}\left|\int_{-\infty}^{t} \alpha_{1} e_{\alpha_{1}}(s, t) \Delta s\right|\right. \\
& \left.+\sup _{t \in[0, \omega]_{\mathbb{T}}}\left|-\frac{k_{2}}{\alpha_{2}} \int_{t}^{+\infty} \ominus \alpha_{2} e_{\ominus \alpha_{2}}(s, t) \Delta s\right|\right) \\
\leq & \left(\left(L_{1}+L_{2}\right)\|\varphi\|_{\mathbb{X}}+a\right)\left(\frac{k_{1}\left(1+\vartheta \alpha_{1}\right)}{\alpha_{1}}+\frac{k_{2}}{\alpha_{2}}\right) .
\end{aligned}
$$

On the other hand, we have

$$
\begin{aligned}
\left|(\Phi \varphi)^{\Delta}\right|_{0}= & \mid\left(\int_{-\infty}^{t} X(t) P X^{-1}(\sigma(s)) f\left(s, \varphi_{s}, \varphi_{s}^{\Delta}\right) \Delta s\right. \\
& \left.-\int_{t}^{+\infty} X(t)(I-P) X^{-1}(\sigma(s)) f\left(s, \varphi_{s}, \varphi_{s}^{\Delta}\right) \Delta s\right)\left._{t}^{\Delta}\right|_{0} \\
= & \mid f\left(t, \varphi_{s}, \varphi_{s}^{\Delta}\right)+A(t)\left(\int_{-\infty}^{t} X(t) P X^{-1}(\sigma(s)) f\left(s, \varphi_{s}, \varphi_{s}^{\Delta}\right) \Delta s\right. \\
& \left.-\int_{t}^{+\infty} X(t)(I-P) X^{-1}(\sigma(s)) f\left(s, \varphi_{s}, \varphi_{s}^{\Delta}\right) \Delta s\right)\left.\right|_{0} \\
\leq & \left(\left(L_{1}+L_{2}\right)\|\varphi\|_{\mathbb{X}}+a\right)\left(\|A\|\left(\frac{k_{1}\left(1+\vartheta \alpha_{1}\right)}{\alpha_{1}}+\frac{k_{2}}{\alpha_{2}}\right)+1\right) .
\end{aligned}
$$


Hence, we have $\|\Phi \varphi\|_{\mathbb{X}} \leq L$, that is, $\Phi \varphi \in \mathbb{X}_{0}$. Next, we show that $\Phi$ is a contraction. For any $\varphi, \psi \in \mathbb{X}_{0}$, we have

$$
\begin{aligned}
|\Phi \varphi-\Phi \psi|_{0}= & \mid \int_{-\infty}^{t} X(t) P X^{-1}(\sigma(s))\left(f\left(s, \varphi_{s}, \varphi_{s}^{\Delta}\right)-f\left(s, \psi_{s}, \psi_{s}^{\Delta}\right)\right) \Delta s \\
& -\left.\int_{t}^{+\infty} X(t)(I-P) X^{-1}(\sigma(s))\left(f\left(s, \varphi_{s}, \varphi_{s}^{\Delta}\right)-f\left(s, \psi_{s}, \psi_{s}^{\Delta}\right)\right) \Delta s\right|_{0} \\
\leq & \left.\sup _{t \in[0, \omega]_{\mathbb{T}}}\left|\int_{-\infty}^{t}\right| X(t) P X^{-1}(\sigma(s))\right|_{0}\left|f\left(s, \varphi_{s}, \varphi_{s}^{\Delta}\right)-f\left(s, \psi_{s}, \psi_{s}^{\Delta}\right)\right|_{0} \Delta s \\
& +\int_{t}^{+\infty}\left|X(t)(I-P) X^{-1}(\sigma(s))\right|_{0}\left|f\left(s, \varphi_{s}, \varphi_{s}^{\Delta}\right)-f\left(s, \psi_{s}, \psi_{s}^{\Delta}\right)\right|_{0} \Delta s \mid \\
\leq & \left(L_{1}+L_{2}\right)\|\varphi-\psi\|_{\mathbb{X}}\left(\frac{k_{1}\left(1+\vartheta \alpha_{1}\right)}{\alpha_{1}}+\frac{k_{2}}{\alpha_{2}}\right)
\end{aligned}
$$

and

$$
\begin{aligned}
\left|(\Phi \varphi-\Phi \psi)^{\Delta}\right|_{0}= & \mid\left[\int_{-\infty}^{t} X(t) P X^{-1}(\sigma(s))\left(f\left(s, \varphi_{s}, \varphi_{s}^{\Delta}\right)-f\left(s, \psi_{s}, \psi_{s}^{\Delta}\right)\right) \Delta s\right. \\
& \left.-\int_{t}^{+\infty} X(t)(I-P) X^{-1}(\sigma(s))\left(f\left(s, \varphi_{s}, \varphi_{s}^{\Delta}\right)-f\left(s, \psi_{s}, \psi_{s}^{\Delta}\right)\right) \Delta s\right]\left._{t}\right|_{0} \\
= & \mid f\left(t, \varphi_{t}, \varphi_{t}^{\Delta}\right)-f\left(t, \psi_{t}, \psi_{t}^{\Delta}\right)+A(t)\left[\int_{-\infty}^{t} X(t) P X^{-1}(\sigma(s))\right. \\
& \times\left(f\left(s, \varphi_{s}, \varphi_{s}^{\Delta}\right)-f\left(s, \psi_{s}, \psi_{s}^{\Delta}\right)\right) \Delta s \\
& \left.-\int_{t}^{+\infty} X(t)(I-P) X^{-1}(\sigma(s))\left(f\left(s, \varphi_{s}, \varphi_{s}^{\Delta}\right)-f\left(s, \psi_{s}, \psi_{s}^{\Delta}\right)\right) \Delta s\right]\left.\right|_{0} \\
\leq & \left(L_{1}+L_{2}\right)\left(\|A\|\left(\frac{k_{1}\left(1+\vartheta \alpha_{1}\right)}{\alpha_{1}}+\frac{k_{2}}{\alpha_{2}}\right)+1\right)\|\varphi-\psi\|_{\mathbb{X}} .
\end{aligned}
$$

By $\left(H_{3}\right)$, we have $\|\Phi \varphi-\Phi \psi\|_{\mathbb{X}} \leq q\|\varphi-\psi\|_{\mathbb{X}}$. It follows that $\Phi$ is a contraction. Therefore, according to the Banach fixed-point theorem, $\Phi$ has a fixed point in $\mathbb{X}_{0}$, that is, (1) has a unique periodic solution in $\mathbb{X}_{0}$. This completes the proof of Theorem 1 .

In view of Lemma 5 and Theorem 1, we have the following corollary:

Corollary 1 Let $\left(H_{1}\right)$ and $\left(H_{3}\right)$ hold. Suppose that

$\left(H_{2}^{\prime}\right)$ there is a constant $\delta>0$ such that

Then (1) has a unique $\omega$-periodic solution.

$$
\left|a_{i i}(t)\right|-\sum_{j \neq i}\left|a_{i j}(t)\right|-\frac{1}{2} \mu(t)\left(\sum_{j=1}^{n}\left|a_{i j}(t)\right|\right)^{2}-\delta^{2} \mu(t) \geq 2 \delta, t \in \mathbb{T}, \quad i=1,2, \ldots, n .
$$

\section{Global exponential stability of periodic solution}

Theorem 2 Let $\left(H_{1}\right)-\left(H_{3}\right)$ hold. Suppose further that 
( $\left.H_{4}\right) \quad L_{1}+L_{2}+\frac{\|A\|}{N}<1$ and $N\left(L_{1}+L_{2}\right)(1+\vartheta)\|A\|<\alpha$. Then the periodic solution of (1) is globally exponentially stable.

Proof By Theorem 1, (1) has an $\omega$-periodic solution $x(t)$ with the initial value $\varphi(s)$. Suppose that $y(t)$ is an arbitrary solution of (1) with the initial value $\psi(s)$. Denote $z(t)=y(t)-x(t)$. Then it follows from (1) that for $t \in \mathbb{T}$,

$$
z^{\Delta}(t)=A(t) z(t)+f\left(t, y_{t}, y_{t}^{\Delta}\right)-f\left(t, x_{t}, x_{t}^{\Delta}\right) .
$$

The initial value condition of (4) is

$$
\phi(s)=\psi(s)-\varphi(s), \phi^{\Delta}(s)=\psi^{\Delta}(s)-\varphi^{\Delta}(s), s \in[-\theta, 0]_{\mathbb{T}} .
$$

By Lemma 1 , for $t_{0} \in[-\theta, 0)_{\mathbb{T}}$ with $t_{0}<t$, we have

$$
z(t)=e_{A}\left(t, t_{0}\right) z\left(t_{0}\right)+\int_{t_{0}}^{t} e_{A}(t, \sigma(s))\left[f\left(s, y_{s}, y_{s}^{\Delta}\right)-f\left(s, x_{s}, x_{s}^{\Delta}\right)\right] \Delta s .
$$

Take a constant $0<\lambda<\alpha$ with $-\lambda \in \mathcal{R}^{+}$and let

$$
M>\max \left\{\frac{N \alpha}{\alpha-N\left(L_{1}+L_{2}\right)(1+\vartheta)\|A\|}, \frac{N \alpha\|A\|}{\alpha-\left(L_{1}+L_{2}\right)\left(\alpha+N(1+\vartheta)\|A\|^{2}\right)}\right\} .
$$

By $\left(H_{4}\right)$, it is easy to verify that $M>1$ and hence, we have

$$
\|z\|_{\mathbb{X}} \leq M\|\phi\|_{\mathbb{X}} e_{\ominus \lambda}\left(t, t_{0}\right), \quad \forall t \in\left[-\theta, t_{0}\right]_{\mathbb{T}} .
$$

We claim that

$$
\|z\|_{\mathbb{X}} \leq M\|\phi\|_{\mathbb{X}} e_{\ominus \lambda}\left(t, t_{0}\right), \quad \forall t \in\left(t_{0},+\infty\right)_{\mathbb{T}} .
$$

To prove this claim, we show that for any constant $p>1$, the following inequality holds

$$
\|z\|_{\mathbb{X}}<p M\|\phi\|_{\mathbb{X}} e_{\ominus \lambda}\left(t, t_{0}\right), \quad \forall t \in\left(t_{0},+\infty\right)_{\mathbb{T}},
$$

which means that

$$
|z|_{0}<p M\|\phi\|_{\mathbb{X}} e_{\ominus \lambda}\left(t, t_{0}\right), \quad \forall t \in\left(t_{0},+\infty\right)_{\mathbb{T}}
$$

and

$$
\left|z^{\Delta}\right|_{0}<p M\|\phi\|_{\mathbb{X}} e_{\ominus \lambda}\left(t, t_{0}\right), \quad \forall t \in\left(t_{0},+\infty\right)_{\mathbb{T}} .
$$

By way of contradiction, assume that (7) does not hold. We will have the following three cases. Case One: (9) is true and (8) is not true. Then there exists $t_{1} \in\left(t_{0},+\infty\right)_{\mathbb{T}}$ such that

$$
|z|_{0} \geq p M\|\phi\|_{\mathbb{X}} e_{\ominus \lambda}\left(t_{1}, t_{0}\right),|z|_{0}<p M\|\phi\|_{\mathbb{X}} e_{\ominus \lambda}\left(t, t_{0}\right), \quad t \in\left(t_{0}, t_{1}\right)_{\mathbb{T}} .
$$

Hence, there must be a constant $c \geq 1$ such that

$$
|z|_{0}=c p M|| \phi\left|\left\|_{\mathbb{X}} e_{\ominus \lambda}\left(t_{1}, t_{0}\right), \quad|z|_{0}<p M|| \phi\right\|_{\mathbb{X}} e_{\ominus \lambda}\left(t, t_{0}\right), \quad t \in\left(t_{0}, t_{1}\right)_{\mathbb{T}} .\right.
$$

Then, by (5), for $t=t_{1}$, we have 


$$
\begin{aligned}
|z|_{0}= & \left|e_{A}\left(t, t_{0}\right) z\left(t_{0}\right)+\int_{t_{0}}^{t} e_{A}(t, \sigma(s))\left[f\left(s, y_{s}, y_{s}^{\Delta}\right)-f\left(s, x_{s}, x_{s}^{\Delta}\right)\right] \Delta s\right|_{0} \\
\leq & \left|e_{A}\left(t, t_{0}\right)\right|_{0}\|\phi\|_{\mathbb{X}}+\left|\int_{t_{0}}^{t} e_{A}(t, \sigma(s))\left[f\left(s, y_{s}, y_{s}^{\Delta}\right)-f\left(s, x_{s}, x_{s}^{\Delta}\right)\right] \Delta s\right|_{0} \\
\leq & \left|e_{A}\left(t, t_{0}\right)\right|_{0}\|\phi\|_{\mathbb{X}}+\left.\sup _{t \in[0, \omega]_{\mathbb{T}}}\left|\int_{t_{0}}^{t}\right| e_{A}(t, \sigma(s))\right|_{0}\left|f\left(s, y_{s}, y_{s}^{\Delta}\right)-f\left(s, x_{s}, x_{s}^{\Delta}\right)\right|_{0} \Delta s \mid \\
\leq & N e_{-\alpha}\left(t_{1}, t_{0}\right)\|\phi\|_{\mathbb{X}}+\left.\left(L_{1}+L_{2}\right)\|z\|_{\mathbb{X}} \sup _{t \in[0, \omega]_{\mathbb{T}}}\left|\int_{t_{0}}^{t}\right| e_{A}(t, \sigma(s))\right|_{0} \Delta s \mid \\
\leq & N e_{\ominus \lambda}\left(t_{1}, t_{0}\right)\|\phi\|_{\mathbb{X}}+\left(L_{1}+L_{2}\right) c p M e_{\ominus \lambda}\left(t_{1}, t_{0}\right) \\
& \times\left.\|\phi\|_{\mathbb{X}} \sup _{t \in[0, \omega] \mathbb{T}_{\mathbb{T}}}\left|\int_{t_{0}}^{t}\right|(I+\mu(s) A(s)) e_{A}(t, s)\right|_{0} \Delta s \mid \\
\leq & N e_{\ominus \lambda}\left(t_{1}, t_{0}\right)\|\phi\|_{\mathbb{X}}+\left(L_{1}+L_{2}\right) c p N M e_{\ominus \lambda}\left(t_{1}, t_{0}\right) \\
& \times\|\phi\|_{\mathbb{X}}(1+\vartheta\|A\|) \sup _{t \in[0, \omega]_{\mathbb{T}}}\left|\int_{t_{0}}^{t} e_{-\alpha}\left(s, t_{0}\right) \Delta s\right| \\
\leq & N e_{\ominus \lambda}\left(t_{1}, t_{0}\right)\|\phi\| \|_{\mathbb{X}}+\frac{\left(L_{1}+L_{2}\right) c p N M e_{\ominus \lambda}\left(t_{1}, t_{0}\right)\|\phi\|_{\mathbb{X}}(1+\vartheta)\|A\|}{\alpha} \\
< & c p M e_{\ominus \lambda}\left(t_{1}, t_{0}\right)\|\phi\|_{\mathbb{X}}\left(\frac{N}{M}+\frac{N\left(L_{1}+L_{2}\right)(1+\vartheta)\|A\|}{\alpha}\right) \\
< & c p M e_{\ominus \lambda}\left(t_{1}, t_{0}\right)\|\phi\|_{\mathbb{X}},
\end{aligned}
$$

which is a contradiction.

Case Two: (8) is true and (9) is not true. Then there exists $t_{2} \in\left(t_{0},+\infty\right)_{\mathbb{T}}$ such that

$$
\left|z^{\Delta}\right|_{0} \geq p M|| \phi\left\|_{\mathbb{X}} e_{\ominus \lambda}\left(t_{2}, t_{0}\right),\left|z^{\Delta}\right|_{0}<p M\right\| \phi \|_{\mathbb{X}} e_{\ominus \lambda}\left(t, t_{0}\right), \quad t \in\left(t_{0}, t_{2}\right)_{\mathbb{T}} .
$$

Hence, there must be a constant $b \geq 1$ such that

$$
\left|z^{\Delta}\right|_{0}=b p M\|\phi\|_{\mathbb{X}} e_{\ominus \lambda}\left(t_{2}, t_{0}\right),\left|z^{\Delta}\right|_{0}<p M\|\phi\|_{\mathbb{X}} e_{\ominus \lambda}\left(t, t_{0}\right), \quad t \in\left(t_{0}, t_{2}\right)_{\mathbb{T}} .
$$

In view of (5), for $t=t_{2}$, we have

$$
\begin{aligned}
\left|z^{\Delta}\right|_{0}= & \mid A(t) e_{A}\left(t, t_{0}\right) z\left(t_{0}\right)+f\left(t, y_{t}, y_{t}^{\Delta}\right)-f\left(t, x_{t}, x_{t}^{\Delta}\right) \\
& +\left.A(t) \int_{t_{0}}^{t} e_{A}(t, \sigma(s))\left(f\left(s, y_{s}, y_{s}^{\Delta}\right)-f\left(s, x_{s}, x_{s}^{\Delta}\right)\right) \Delta s\right|_{0} \\
\leq & \left.\|A\||| e_{A}\left(t, t_{0}\right)\right|_{0}\|\phi\|_{\mathbb{X}}+\left|f\left(t, y_{t}, y_{t}^{\Delta}\right)-f\left(t, x_{t}, x_{t}^{\Delta}\right)\right|_{0} \\
& +\left|A(t) \int_{t_{0}}^{t} e_{A}(t, \sigma(s))\left(f\left(s, y_{s}, y_{s}^{\Delta}\right)-f\left(s, x_{s}, x_{s}^{\Delta}\right)\right) \Delta s\right|_{0} \\
\leq & \|A\| N e_{-\alpha}\left(t_{2}, t_{0}\right)\left|\phi\left\|_{\mathbb{X}}+\left(L_{1}+L_{2}\right) b p M e_{\ominus \lambda}\left(t_{2}, t_{0}\right) \mid \phi\right\|_{\mathbb{X}}\right. \\
& +\frac{\|A\|^{2} N b p M\left(L_{1}+L_{2}\right) e_{\ominus \lambda}\left(t_{2}, t_{0}\right) \mid \phi \|_{\mathbb{X}}(1+\vartheta)}{\alpha} \\
< & b p M e_{\ominus \lambda}\left(t_{2}, t_{0}\right) \mid \phi \|_{\mathbb{X}}\left(\frac{N\|A\|}{M}+L_{1}+L_{2}+\frac{\|A\|^{2} N\left(L_{1}+L_{2}\right)(1+\vartheta)}{\alpha}\right) \\
< & b p M e_{\ominus \lambda}\left(t_{2}, t_{0}\right) \mid \phi \|_{\mathbb{X}},
\end{aligned}
$$


which is also a contradiction.

Case Three: (8) and (9) are both untrue. By Case One and Case Two, we can obtain a contradiction. Therefore, (7) holds. Let $p \rightarrow 1$, (6) holds. Hence, we have that

$$
\|y-x\|_{\mathbb{X}} \leq M\|\varphi-\psi\|_{\mathbb{X}} e_{\ominus \lambda}\left(t, t_{0}\right), \quad t \in[-\theta, \infty)_{\mathbb{T}}, t \geq t_{0},
$$

which implies that the periodic solution $x(t)$ of (1) is globally exponentially stable. This completes the proof of Theorem 2.

Corollary 2 Let $\left(H_{1}\right),\left(H_{2}^{\prime}\right)$ and $\left(H_{3}\right)-\left(H_{5}\right)$ hold. Then (1) has a unique periodic solution, which is globally exponentially stable.

\section{An example}

In (1), if we take

$$
A(t)=\left(\begin{array}{cc}
-0.002 \sin 2 t & 0 \\
0 & -0.001 \cos 2 t
\end{array}\right), \quad f=\left(f_{1}, f_{2}\right)^{T},
$$

where

$$
\begin{aligned}
& f_{1}\left(t, \varphi, \varphi^{\Delta}\right)=0.0003\left(\sin \varphi(-\tau(t))+\cos \varphi^{\Delta}(-\zeta(t))\right), \\
& f_{2}\left(t, \varphi, \varphi^{\Delta}\right)=0.0002\left(\sin \varphi(-\tau(t))+\cos \varphi^{\Delta}(-\zeta(t))\right),
\end{aligned}
$$

$\tau, \zeta \in C\left(\mathbb{T}, \mathbb{T} \cap \mathbb{R}^{+}\right)$are $\pi$-periodic. Then (1) reduces to

$$
\begin{aligned}
\left(\begin{array}{l}
x^{\Delta}(t) \\
x^{\Delta}(t)
\end{array}\right)= & \left(\begin{array}{cc}
-0.002 \sin 2 t & 0 \\
0 & -0.001 \cos 2 t
\end{array}\right)\left(\begin{array}{l}
x_{1}(t) \\
x_{2}(t)
\end{array}\right) \\
& +\left(\begin{array}{l}
0.0003\left(\sin \varphi(-\tau(t))+\cos \varphi^{\Delta}(-\zeta(t))\right) \\
0.0002\left(\sin \varphi(-\tau(t))+\cos \varphi^{\Delta}(-\zeta(t))\right)
\end{array}\right) .
\end{aligned}
$$

By a simple calculation, we have $L_{1}=L_{2}=0.0003,\|A\|=0.002, \ominus \alpha_{1}=-0.001$, $k_{1}=1, \ominus \alpha_{2}=k_{2}=0, \alpha=0.001$. One can easily verify that all the conditions in Corollary 2 are satisfied for $0 \leq \mu \leq 1$. In particularly, if we take $\mathbb{T}=\mathbb{R}$, then $\mu(t)=0$ and if we take $\mathbb{T}=\mathbb{Z}$, then $\mu(t)=1$. Therefore, in both the cases of $\mathbb{T}=\mathbb{R}$ and $\mathbb{T}=\mathbb{Z}$, (10) has a $\pi$-periodic solution, which is exponentially stable.

Remark 4 Example (10) shows that both the continuous case of (10)

$$
\frac{\mathrm{d}}{\mathrm{d} t} x(t)=A(t) x(t)+f\left(t, x(t-\tau(t)), x^{\prime}(t-\zeta(t))\right), \quad t \in \mathbb{R}
$$

and its discrete analogue

$$
\Delta x(n)=A(n) x(n)+f(n, x(n-\tau(n)), \Delta x(n-\zeta(n))), \quad n \in \mathbb{Z}
$$

have the same dynamical property for the periodic case. 


\section{Conclusion}

In this paper, by using the existence of the exponential dichotomy of linear dynamic equations on time scales and the inequality techniques, we established the existence and global exponential stability of periodic solutions for a very general class of $n$-dimensional neutral dynamic equations on time scales. Our results of this paper are completely new and complementary to the previously known results even in both the case of differential equations (time scale $\mathbb{T}=\mathbb{R}$ ) and the case of difference equations (time scale $\mathbb{T}=\mathbb{Z}$ ), and our methods used in this paper may be used to study the problem of periodic solutions to other types of dynamic equations on time scales.

Authors' contributions

$\mathrm{BL}, \mathrm{YL}$ and $\mathrm{XZ}$ contributed equally to the manuscript and typed. All authors read and approved the final manuscript.

\section{Author details}

${ }^{1}$ Department of Mathematics, Yunnan University, Kunming 650091, Yunnan, People's Republic of China. ${ }^{2}$ School of Mathamatics and Information Science, Qujing Normal University, Qujing 655011, Yunnan, People's Republic of China.

\section{Acknowledgements}

This work is supported by the National Natural Sciences Foundation of People's Republic of China under Grant 11361072.

\section{Competing interests}

The authors declare that they have no competing interests.

Received: 16 October 2015 Accepted: 16 February 2016

Published online: 29 February 2016

References

Abbas S, Bahuguna D (2008) Almost periodic solutions of neutral functional differential equations. Comput Math Appl 55:2593-2601

Ardjouni A, Djoudi A (2012) Existence of periodic solutions for nonlinear neutral dynamic equations with variable delay on a time scale. Commun Nonlinear Sci Numer Simul 17:3061-3069

Bohner M, Peterson A (2001) Dynamic equations on time scales, an introduction with applications. Birkhäuser, Boston

Chen XX, Lin FX (2010) Almost periodic solutions of neutral functional differential equations. Nonlinear Anal Real World Appl 11:1182-1189

DaCunha JJ (2005) Stability for time varying linear dynamic systems on time scales. J Comput Appl Math 176:381-410

Du NH, Tien LH (2007) On the exponential stability of dynamic equations on time scales. J Math Anal Appl 331:1159-1174

Graef JR, Hill M (2015) Nonoscillation of all solutions of a higher order nonlinear delay dynamic equation on time scales. J Math Anal Appl 423:1693-1703

Hilger S (1990) Analysis on measure chains - a unified approach to continuous and discrete calculus. Results Math 18:18-56

Hovhannisyan G (2014) Asymptotic behavior of a planar dynamic system. Rocky Mountain J Math 44:1203-1242

Kaufmann ER, Raffoul YN (2006) Periodic solutions for a neutral nonlinear dynamical equations on a time scale. J Math Anal Appl 319:315-325

Li TX, Saker SH (2014) A note on oscillation criteria for second-order neutral dynamic equations on isolated time scales. Commun Nonlinear Sci Numer Simul 19:4185-4188

Li YK, Sun LJ (2013) Infinite many positive solutions for nonlinear first-order BVPs with integral boundary conditions on time scales. Topol Methods Nonlinear Anal 41:305-321

Li YK, Xu EL (2011) Three positive periodic solutions for dynamic equations with piecewise constant argument and impulse on time scales. Glasg Math J 53:369-377

Li YK, Zhao L, Chen X (2012) Existence of periodic solutions for neutral type cellular neural networks with delays. Appl Math Model 36:1173-1183

Liu P, Li YK (2004) Positive periodic solutions of infinite delay functional differential equations depending on a parameter. Appl Math Comput 150:159-168

Lupulescu V, Younus A (2011) On controllability and observability for a class of linear impulsive dynamic systems on time scales. Math Comput Model 54:1300-1310

Su YH, Feng ZS (2014) Homoclinic orbits and periodic solutions for a class of Hamiltonian systems on time scales. J Math Anal Appl 411:37-62

Wang C, Li YK, Fei Y (2010) Three positive periodic solutions to nonlinear neutral functional differential equations with impulses and parameters on time scales. Math Comput Model 52:1451-1462

Xu DY, Yang ZG, Yang ZC (2007) Exponential stability of nonlinear impulsive neutral differential equations with delays. Nonlinear Anal 67:1426-1439

Zhang ZG, Dong WL, Li QL, Liang HY (2009) Positive solutions for higher order nonlinear neutral dynamic equations on time scales. Appl Math Model 33:2455-2463 
Zhang JM, Fan M, Zhu HP (2010a) Existence and roughness of exponential dichotomies of linear dynamic equations on time scales. Comput Math Appl 59:2658-2675

Zhang JM, Fan M, Zhu HP (2010b) Necessary and sufficient criteria for the existence of exponential dichotomy on time scales. Comput Math Appl 60:2387-2398

Zhang C, Wang LL, Fan YH (2014) Existence of positive solutions for a dynamic equation on measure chains. Appl Math Lett 35:24-28

Zhou JW, Li YK (2010) Sobolev's spaces on time scales and its applications to a class of second order Hamiltonian systems on time scales. Nonlinear Anal 73:1375-1388

Zhou JW, Li YK (2012) Variational approach to a class of second order Hamiltonian systems on time scales. Acta Appl Math 117:47-69

Submit your manuscript to a SpringerOpen ${ }^{\circ}$ journal and benefit from:

- Convenient online submission

- Rigorous peer review

- Immediate publication on acceptance

- Open access: articles freely available online

- High visibility within the field

- Retaining the copyright to your article

Submit your next manuscript at $\mathbf{s p r i n g e r o p e n . c o m ~}$ 